\title{
Metallic Graphene Nanoribbons
}

Cite as

Nano-Micro Lett.

(2021) 13:53

Received: 12 October 2020

Accepted: 9 November 2020

Published online: 5 January 2021

(C) The Author(s) 2021
Sheng-Yi Xie ${ }^{1}$, Xian-Bin $\mathrm{Li}^{2}{ }^{凶}$

ABSTRACT Isolated graphene nanoribbons (GNRs) usually have energy gaps, which scale with their widths, owing to the lateral quantum confinement effect of GNRs. The absence of metallic GNRs limits their applications in device interconnects or being one-dimensional physics platform to research amazing properties based on metallicity. A recent study published in Science provided a novel method to produce metallic GNRs by inserting a symmetric superlattice into other semiconductive GNRs. This finding will broader the applications of GNRs both in nanoelectronics and fundamental science.

KEYWORDS Graphene; Nanoribbons; Quantum confinement effect; Supperlattice

Unlike the gapless semimetal of graphene [1], the graphene nanoribbons (GNRs) [2], whether armchair or zigzag type, usually own an energy gap scaling inversely with their widths due to the lateral quantum confinement effect of GNRs [3]. The raised energy gap, which is absent in graphene, enables the production of transistor [4], yet the robust semicondutivity of GNRs limits the applications in such as device interconnects or being one-dimensional physics platform to explore superconductivity [5], Luttinger liquid [6], charge density waves [7] or spintronics [8]. Recently, one paper published in Science reported an ingenious method to produce metallic GNRs [9] based on the atomically precise bottom-up synthesis.

In this work, Rizzo et al. in University of California at Berkeley used the precursor molecule 1 (Fig. 1a) to construct the GNRs with the symmetrical insertion of methyl groups to form the superlattice, which are named as the sawtooth GNR (sGNR). Upon annealing over $350{ }^{\circ} \mathrm{C}$, the sGNRs transformed to the called five sawtooth sGNRs (5-sGNRs) with minor chemical bond rearrangements to form a fivemembered ring along their edges, as also shown in Fig. 1a. Electronic structures of sGNRs and 5-sGNRs were further determined both by scanning tunneling microscope (STM) spectroscopy and density functional theory (DFT) calculations. The experimental $\mathrm{d} I / \mathrm{d} V$ point spectrum of a sGNR is shown in Fig. 1b, and the sharp peak states as well as their projection in real space (Fig. 1c) at the zero bias clearly show the metallic density of states (DOS), which agrees well with the DFT results (Fig. 1d, e). In their further experiment, the DOS of 5-sGNRs spans a broader energy range around the Fermi level, inducing the robust metallicity with a 20 -fold increase of the metallic bandwidth, as shown in Fig. 1.

This work provides a smart strategy for realizing the metallicity in GNRs to act as a candidate used in logic devices. In future, as a direct measurement of metallicity, variable temperature conductivity experiments can be further considered. Additionally, the performance comparison between these metallic GNRs and traditional metals like copper used in interconnect technology is also meaningful for the applications in nanoelectronics. Finally, considering the possible formation of junctions between these metallic GNRs and ordinary semiconductive GNRs, whether Ohmic contact or Schottky contact can be formed should also deserve extra efforts.

Xian-Bin Li, lixianbin@jlu.edu.cn

School of Physics and Electronics, Hunan University, 410082 Changsha, People's Republic of China

2 State Key Laboratory of Integrated Optoelectronics, College of Electronic Science and Engineering, Jilin University, 130012 Changchun, People's Republic of China 


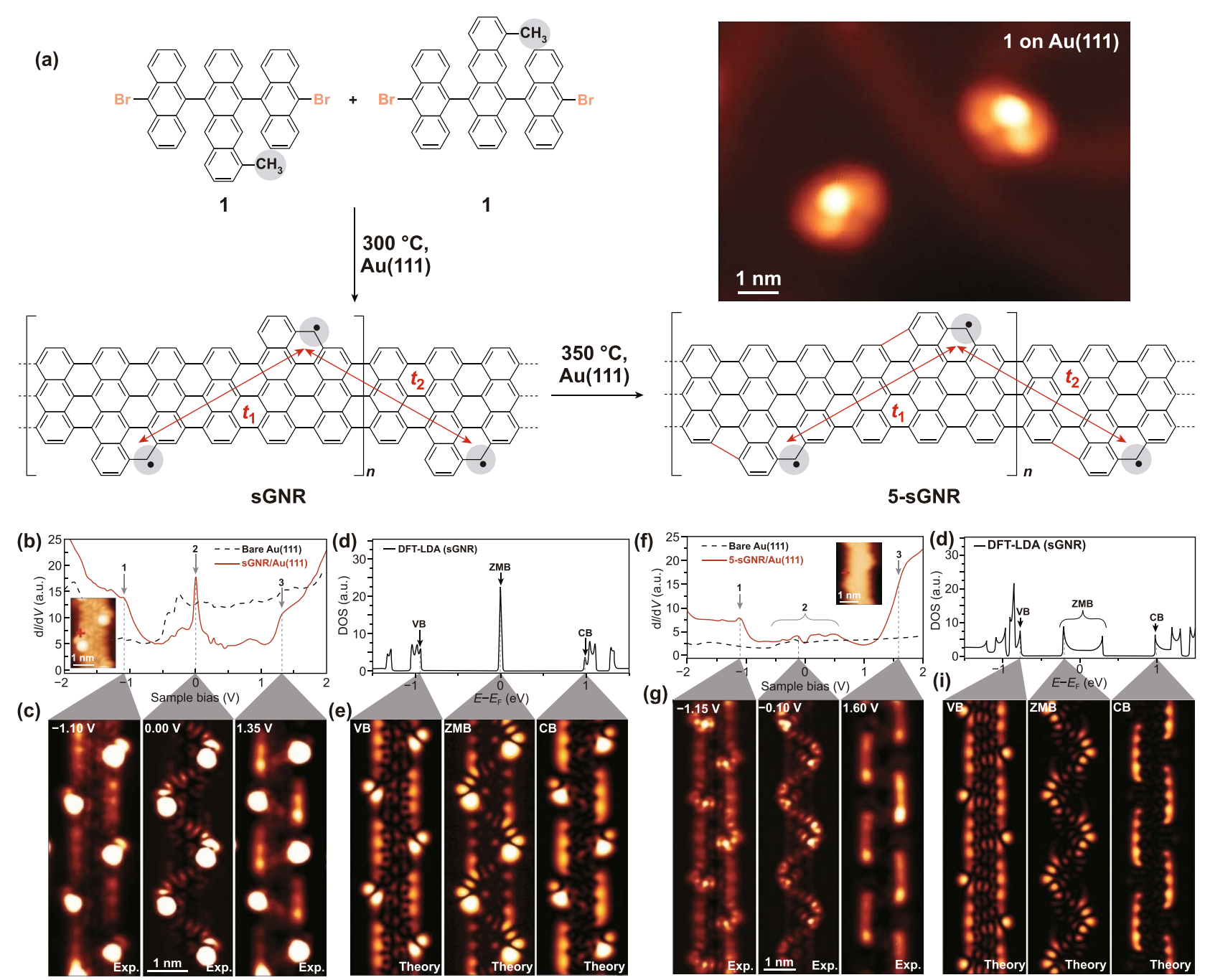

Fig. 1 a Schematic bottom-up growth of sGNRs and 5-sCNRs from molecular precursor 1 on Au (111), and the inset at the top right corner shows STM topograph of two isolated monomers of precursor 1. b-e Electronic structure of sGNRs. b experimental $\mathrm{d} I / \mathrm{d} V$ point spectroscopy, $\mathbf{c}$ related constant-height $\mathrm{d} I / \mathrm{d} V$ maps in real space, $\mathbf{d}$ DFT-LDA calculated DOS, and e related local DOS maps of a sGNR. f-i Electronic structure of 5-sCNRs. f experimental $\mathrm{d} I / \mathrm{d} V$ point spectroscopy, $\mathbf{g}$ related constant-height $\mathrm{d} I / \mathrm{d} V$ maps, $\mathbf{h}$ DFT-LDA calculated DOS, and $\mathbf{i}$ related local DOS maps of a 5-sCNR. Reproduced from Ref. [9]. Copyright 2020 American Association for the Advancement of Science.

Open Access This article is licensed under a Creative Commons Attribution 4.0 International License, which permits use, sharing, adaptation, distribution and reproduction in any medium or format, as long as you give appropriate credit to the original author(s) and the source, provide a link to the Creative Commons licence, and indicate if changes were made. The images or other third party material in this article are included in the article's Creative Commons licence, unless indicated otherwise in a credit line to the material. If material is not included in the article's Creative Commons licence and your intended use is not permitted by statutory regulation or exceeds the permitted use, you will need to obtain permission directly from the copyright holder. To view a copy of this licence, visit http://creativecommons.org/licenses/by/4.0/.

\section{References}

1. K.S. Novoselov, A.K. Geim, S.V. Morozov, D. Jiang, M.I. Katsnelson et al., Two-dimensional gas of massless dirac fermions in graphene. Nature 438(7065), 197-200 (2005). https ://doi.org/10.1038/nature04233

2. J.M. Cai, P. Ruffieux, R. Jaafar, M. Bieri, T. Braun et al., Atomically precise bottom-up fabrication of graphene nanoribbons. Nature 466(7305), 470-473 (2010). https://doi. org/10.1038/nature09211

3. Y.W. Son, M.L. Cohen, S.G. Louie, Energy gaps in graphene nanoribbons. Phys. Rev. Lett. 97(21), 216803 (2006). https:// doi.org/10.1103/PhysRevLett.97.216803 
4. B. Obradovic, R. Kotlyar, F. Heinz, P. Matagne, T. Rakshit et al., Analysis of graphene nanoribbons as a channel material for field-effect transistors. Appl. Phys. Lett. 88(14), 142102 (2006). https://doi.org/10.1063/1.2191420

5. I. Takesue, J. Haruyama, N. Kobayashi, S. Chiashi, S. Maruyama et al., Superconductivity in entirely end-bonded multiwalled carbon nanotubes. Phys. Rev. Lett. 96(5), 057001 (2006). https://doi.org/10.1103/PhysRevLett.96.057001

6. M. Bockrath, D.H. Cobden, J. Lu, A.G. Rinzler, R.E. Smalley et al., Luttinger-liquid behaviour in carbon nanotubes. Nature 397(6720), 598-601 (1999). https://doi.org/10.1038/17569
7. G. Grüner, A. Zawadowski, P.M. Chaikin, Nonlinear conductivity and noise due to charge-density-wave depinning in $\mathrm{NbSe}_{3}$. Phys Rev Lett. 46(7), 511-515 (1981). https://doi. org/10.1103/PhysRevLett.46.511

8. Y. Liu, C. Zeng, J. Zhong, J. Ding, Z.M. Wang et al., Spintronics in two-dimensional materials. Nano-Micro. Lett. 12(1), 93 (2020). https://doi.org/10.1007/s40820-020-00424-2

9. D.J. Rizzo, G. Veber, J. Jiang, R. McCurdy, T. Cao et al., Inducing metallicity in graphene nanoribbons via zero-mode superlattices. Science 369(6511), 1597-1603 (2020). https:// doi.org/10.1126/science.aay3588 